Megha Prasad, MD MS

Columbia University Medical Center,

Department of Medicine, New York City, NY
Kavel Visrodia, MD

Massachusetts General Hospital,

Department of Medicine, Boston, MA

\title{
Should I prone non-ventilated awake patients with COVID-19?
}

\section{Posted June 19, 2020}

\section{ABSTRACT}

The initial evidence supporting the prone position in non-ventilated awake patients with COVID-19 infection was anecdotal and theoretical. However, several early studies support the potential role of this practice to improve oxygenation, improve clinical outcomes, and reduce the need for intensive care admission. Although there is not a set amount of time that has been proven to be optimal for awake proning, we recommend sessions of 2 to 3 hours for at least a total 4 to 5 hours a day as tolerated based on the existing data and our anecdotal experience. Despite the low-risk and low-cost nature of proning awake patients, there are still logistical concerns that may prevent adequate proning, the most significant of which is patient comfort and compliance.

\section{INTRODUCTION}

Prone positioning is a well-established and routine intervention for patients with moderate-to-severe acute respiratory distress syndrome (ARDS) who require mechanical ventilation. ${ }^{1}$ As COVID-19 infection spread and evolved into a global pandemic, anecdotal evidence also suggested a role for proning of non-ventilated, awake patients with COVID-19 infection. ${ }^{2}$ Despite rapidly evolving research and novel therapeutics, COVID-19 infection remains associated with significant morbidity and mortality, especially in the setting of critical illness, leading to multi-organ involvement and shock. ${ }^{2}$ Moreover, limited hospital resources and restrictions on using noninvasive ventilation due to concerns for aerosol-

The statements and opinions expressed in COVID-19 Curbside Consults are based on experience and the available literature as of the date posted. While we try to regularly update this content, any offered recommendations cannot be substituted for the clinical judgment of clinicians caring for individual patients.

The authors report no potential conflicts of interest.

doi:10.3949/ccjm.87a.ccc050 ization may limit the ability of health care providers to quickly mechanically ventilate these patients, making low-risk temporizing measures especially attractive. $^{3}$

Proning non-ventilated, awake patients, has been proposed as a potential low-risk intervention that may be associated with improved oxygenation, clinical outcomes, and reduced need for intensive care admission. Several studies since the beginning of the pandemic have addressed the potential role of proning awake patients with COVID-19 infection in optimizing outcomes.

\section{PHYSIOLOGY OF PRONING IN ACUTE HYPOXEMIC RESPIRATORY FAILURE}

Prone positioning may affect the mechanics and physiology of gas exchange to improve oxygenation in both intubated and non-intubated patients, and may be even more effective in the latter. ${ }^{4,5}$ Prone positioning may reduce the difference between ventral and dorsal pleural pressure, or the "ventral-dorsal transpulmonary difference." Alveoli that collapse during supine positioning may be recruited over time with prone positioning and improve oxygenation. On the other hand, supine positioning is associated with a higher dorsal than ventral pleural pressure, causing greater ventral alveoli expansion. ${ }^{4}$

Additionally, supine positioning may be associated with compression of the medial posterior lung parenchyma and diaphragm. During positioning, the heart becomes dependent, decreasing posterior medial lung compression. This may shift perfusion toward healthier alveoli in the anterior lungs, allowing for improved ventilation-perfusion matching and reduced intrapulmonary shunting. Lastly, proning may improve secretion management, which can, in turn, prevent atelectasis and improve recruitment of pulmonary parenchyma. ${ }^{4}$ 
TABLE 1

Awake proning in patients with COVID-19 infection

\begin{tabular}{|c|c|c|c|}
\hline Study & $\begin{array}{l}\text { No. of } \\
\text { patients }\end{array}$ & Protocol & Findings \\
\hline Elharrar et $\mathrm{al}^{6}$ & 24 & $\begin{array}{l}\text { As tolerated, } \\
\text { majority }>3 \text { hours }\end{array}$ & $\begin{array}{l}\text { Oxygenation improvement } \\
\text { in a subset of patients }\end{array}$ \\
\hline Sartini et $\mathrm{al}^{7}$ & 15 & $\begin{array}{l}\text { Noninvasive ventilation } \\
\text { in addition to proning } \\
\text { for median of } 2 \text { times, } \\
3 \text { hours each per day }\end{array}$ & Improved oxygenation \\
\hline $\mathrm{Ng}$ et $\mathrm{al}^{8}$ & 10 & $\begin{array}{l}1 \text { hour each, } 5 \text { sessions } \\
\text { a day, spaced } 3 \text { hours } \\
\text { apart }\end{array}$ & $\begin{array}{l}\text { Improved oxygenation and } \\
\text { reduced need for mechanical } \\
\text { ventilation }\end{array}$ \\
\hline Caputo et al ${ }^{9}$ & 50 & 5 minutes of proning & $\begin{array}{l}\text { Improvement in oxygenation } \\
\text { with proning }\end{array}$ \\
\hline
\end{tabular}

oxygen saturation with only one-third of patients requiring intubation. ${ }^{9}$

Due to limited data on its efficacy, there is not a set amount of time that has been proven to be optimal for awake proning, with each study employing slightly different protocols largely dependent on patient tolerance. ${ }^{2}$ For example, some protocols call for patients to rotate positions every 2 hours, starting with left lateral recumbent, right lateral recumbent, sitting upright 60 to 90 degrees, and lastly, lying prone in bed. Others have suggested at least 2 separate 3 -hour sessions of proning per day. Based on the existing data and our anecdotal experience, we recommend sessions of 2 to 3 hours for at least a total 4 to 5 hours a day as tolerated. $2,6,8,10,11$

Thus several studies have suggested

\section{THE EVIDENCE}

Initially, the evidence supporting the prone position in awake patients with COVID-19 infection was anecdotal and theoretical based on principles applied from proning of mechanically ventilated patients and studies of non-COVID patients, but there has since been several early studies supporting the potential role of this practice (Table 1). ${ }^{6-9}$ These studies are inherently limited, however, by their small size and lack of controls, but larger robust studies are in progress.

Several case reports and small case series initially noted a potential improvement in oxygenation with awake proning in patients with COVID-19 infection. Elharrar et al reported in a single-center study of 24 patients that prone positioning was associated with an increase in $\mathrm{PaO} 2$ of more than $20 \%$ from baseline. ${ }^{6}$ Several of these patients, however, did not tolerate proning or did not have sustained improvement in oxygenation once turned supine.

Sartini et al in a cross-sectional study of 15 awake patients with mild and moderate ARDS noted a sustained improvement in most patients in conjunction with noninvasive ventilation. ${ }^{7}$

$\mathrm{Ng}$ et al noted a reduced need for mechanical ventilation and improved outcomes in a series of patients who underwent prone positioning for 1 hour at a time, 5 times a day spaced 3 hours apart. ${ }^{8}$ Caputo et al studied early safe proning with up to 120 minutes as tolerated in 50 awake non-intubated patients with refractory hypoxemia (median oxygen saturation of $84 \%$ ) and noted an immediate increase in median a potential benefit of proning awake patients with COVID-19 infection, but these studies are small and limited in their scope. Despite the low-risk and lowcost nature of proning awake patients, there are still logistical concerns that may prevent adequate proning, the most significant of which is patient comfort and compliance.

\section{LOGISTICAL BENEFITS OF PRONE POSITIONING AND POTENTIAL RISKS}

Initially, early outcomes data suggested a role of early mechanical ventilation in patients with COVID-19 infection. While this approach was adopted in New York City hospitals at the epicenter of the United States COVID-19 outbreak, resource limitations and exceedingly high mortality rates in intubated patients prompted clinicians to explore other experimental strategies to temporize patients and reduce the need for mechanical ventilation.

Prone positioning and its potential efficacy is an especially important consideration in patients with COVID-19 infection during this global pandemic where many institutions and cities have struggled with limited resources. Maneuvers such as awake proning may play a key role in safely improving oxygenation without the need for additional resources. Several studies have described at least temporary improvements in oxygenation, but whether this benefit is sustained even after a patient is supine remains unclear. ${ }^{6,8,11}$

Furthermore, in patients with hypoxemic respira- 
tory failure, noninvasive ventilation may address hypoxemic respiratory failure and prevent the need for mechanical ventilation. However, the implementation of such temporizing measures may be precluded by concerns for aerosolization and the lack of negative pressure rooms. In such patients, prone positioning may especially be important in increasing oxygenation and preventing the need for mechanical ventilation. Larger studies are necessary to better understand the potential benefits of prone positioning in patients with COVID-19 infection.

There are some important contraindications including spinal instability, facial or pelvic fractures, and an open chest or unstable chest wall as well as relative contraindications such as delirium and recent vomiting that should be considered. ${ }^{10}$ Potential risks to consider include disconnection of oxygen supply and gastroesophageal reflux disease/esophageal disease that may develop or be exacerbated by prone positioning. Aside from these considerations, most patients admitted with COVID-19 infection should be considered for awake proning given its limited risks and cost.

\section{CONCLUSIONS}

Although proning awake patients was not routinely used in the beginning of the coronavirus pandemic, recent data suggests potential improvement with this non-invasive, low-risk practice. While the benefit may not be sustained, the practice of proning awake patients with severe hypoxemic respiratory stress may help minimize the need for mechanical ventilation in some patients, and temporize critically ill patients prior to mechanical ventilation. Patient tolerance and compliance may be limitations of awake proning, however. Additional robust randomized trials and physiologic studies are ongoing to address the potential clinical benefits in patients with COVID19 infection. While we await these results, the potential benefit paired with the low risk of proning should encourage clinicians caring for patients with hypoxemic respiratory failure and COVID-19 infection to consider early proning.

\section{REFERENCES}

1. Pelosi P, Brazzi L, Gattinoni L. Prone position in acute respiratory distress syndrome. Eur Respir J 2002; 20(4):1017-1028. doi:10.1183/ 09031936.02.00401702

2. Goyal P, Choi JJ, Pinheiro LC, et al. Clinical characteristics of Covid19 in New York City [published online ahead of print April 17, 2020]. N Engl J Med 2020; NEJMc2010419. doi:10.1056/NEJMc2010419

3. Ran L, Chen X, Wang Y, Wu W, Zhang L, Tan X. Risk factors of healthcare workers with corona virus disease 2019: a retrospective cohort study in a designated hospital of Wuhan in China [published online ahead of print March 17, 2020]. Clin Infect Dis 2020; ciaa287. doi:10.1093/cid/ciaa287

4. Sud S, Sud M, Friedrich JO, Adhikari NK. Effect of prone positioning in patients with acute respiratory distress syndrome and high Simplified Acute Physiology Score II. Crit Care Med 2008; 36(9):27112713. doi:10.1097/CCM.0b013e3181846fc0

5. Valter C, Christensen AM, Tollund C, Schønemann NK. Response to the prone position in spontaneously breathing patients with hypoxemic respiratory failure. Acta Anaesthesiol Scand 2003; 47(4):416-418. doi:10.1034/j.1399-6576.2003.00088.x

6. Elharrar X, Trigui Y, Dols AM, et al. Use of prone positioning in nonintubated patients with COVID-19 and hypoxemic acute respiratory failure [published online ahead of print May 15, 2020]. JAMA 2020; e208255. doi:10.1001/jama.2020.8255

7. Sartini C, Tresoldi M, Scarpellini $\mathbf{P}$, et al. Respiratory parameters in patients with COVID-19 after using noninvasive ventilation in the prone position outside the intensive care unit [published online ahead of print May 15, 2020]. JAMA 2020; e207861. doi:10.1001/ jama.2020.7861

8. Ng Z, Tay WC, Ho CHB. Awake prone positioning for non-intubated oxygen dependent COVID-19 pneumonia patients [published online ahead of print May 26, 2020]. Eur Respir J 2020; 2001198. doi:10.1183/13993003.01198-2020

9. Caputo ND, Strayer RJ, Levitan R. Early self-proning in awake, non-intubated patients in the emergency department: a single ED's experience during the COVID-19 pandemic. Acad Emerg Med 2020; 27(5):375-378. doi:10.1111/acem.139942020.

10. Jiang LG, LeBaron J, Bodnar D, et al. "Conscious proning: an introduction of a proning protocol for non-intubated, awake, hypoxic Emergency Department COVID-19 patients" [published online ahead of print May 27, 2020]. Acad Emerg Med 2020; 10.1111/ acem.14035. doi:10.1111/acem.14035

11. Slessarev M, Cheng J, Ondrejicka M, Arntfield R; Critical Care Western Research Group. Patient self-proning with high-flow nasal cannula improves oxygenation in COVID-19 pneumonia [published online ahead of print April 21, 2020]. Can J Anaesth 2020; 1-3. doi:10.1007/s12630-020-01661-0 